\title{
Acoustic Monitoring of Anomaly Stressed Zones, Determination Their Positions, Surfaces, Evaluation of Catastrophic Risk
}

\author{
Olga Hachay ${ }^{1}$, Oleg Khachay ${ }^{2}$ \\ ${ }^{1}$ Institute of Geophysics, Ural Branch of Russian Academy of Sciences, Yekaterinburg, Russian Federation \\ ${ }^{2}$ Ural Federal University, Yekaterinburg, Russian Federation \\ Email: olgakhachay@yandex.ru, khachay@yandex.ru
}

How to cite this paper: Hachay, $O$. and Khachay, O. (2020) Acoustic Monitoring of Anomaly Stressed Zones, Determination Their Positions, Surfaces, Evaluation of Catastrophic Risk. Open Journal of Geology, 10, 501-509.

https://doi.org/10.4236/ojg.2020.105022

Received: April 9, 2020

Accepted: May 9, 2020

Published: May 12, 2020

Copyright $\odot 2020$ by author(s) and Scientific Research Publishing Inc. This work is licensed under the Creative Commons Attribution International License (CC BY 4.0).

http://creativecommons.org/licenses/by/4.0/

\begin{abstract}
Abstract Problem statement. Self-organization is not a universal property of matter, it exists under certain internal and external conditions and this is not associated with a special class of substances. The study of the morphology and dynamics of migration of anomalous zones associated with increased stresses is of particular importance in the development of deep deposits, complicated by dynamic phenomena in the form of rock impacts. Applied method and design: An important tool for this study is geophysical exploration. To describe the geological environment in the form of an array of rocks with its natural and technogenic heterogeneity, one should use its more adequate description, which is a discrete model of the medium in the form of a piecewise heterogeneous block medium with embedded heterogeneities of a lower rank than the block size. This nesting can be traced several times, i.e. changing the scale of the study; we see that heterogeneities of a lower rank now appear in the form of blocks for heterogeneities of the next rank. A simple averaging of the measured geophysical parameters can lead to distorted ideas about the structure of the medium and its evolution. Typical results: We have analyzed the morphology of the structural features of disintegration zones before a strong dynamic phenomenon. The introduction of the proposed integrated passive and active geophysical monitoring into the mining system, aimed at studying the transient processes of the redistribution of stress-strain and phase states, can help prevent catastrophic dynamic manifestations during the development of deep-located deposits. Concluding note (Practical value/implications): Active geophysical monitoring methods should be tuned to a model of a hierarchical heterogeneous environment. Iterative algorithms for $2-\mathrm{D}$ modeling and interpretation for sound diffraction and a linearly polarized transverse elastic wave on the inclusion with a
\end{abstract}


hierarchical elastic structure located in the J-th layer of the N-layer elastic medium are constructed.

\section{Keywords}

High-Stressed Zones, Electromagnetic Induction Method, Acoustic Method, Hierarchical Inclusions, Layered-Block Medium, Algorithms of Modeling And Interpretation, Estimation of Massif No Stability

\section{Introduction}

The formation of structures during irreversible processes is associated with a qualitative leap when threshold (critical) parameters are reached. Self-organization is a supercritical phenomenon when the parameters of the system exceed their critical values. When a system deviates strongly from an equilibrium state, its variables satisfy non-linear equations. Nonlinearity is an important and general feature of processes that occur far from equilibrium. Moreover, the supercritical return of entropy is possible only if there is an unusual, special internal structure of the system [1]. This means that self-organization is not a universal property of matter, it exists under certain internal and external conditions and this is not associated with a special class of substances. So, there are two classes of irreversible processes: 1) destruction of the structure near the equilibrium position, this is a universal property of systems under arbitrary conditions; 2) the appearance of structures far from the equilibrium position under the conditions that the system is open and has nonlinear internal dynamics, and its external parameters have supercritical values. I. Prigogine called them dissipative structures [2]. The study of the morphology and dynamics of migration of these zones (named as disintegration zones) is of particular importance in the development of deep deposits, complicated by dynamic phenomena in the form of mountain impacts. An important tool for this study is geophysical exploration. As shown in [3], to describe the geological environment in the form of an array of rocks with its natural and technogenic heterogeneity, one should use its more adequate description, which is a discrete model of the medium in the form of a piecewise inhomogeneous block medium with embedded heterogeneities of lower rank than the block size. This nesting can be traced several times, i.e. changing the scale of the study; we see that heterogeneities of a lower rank now appear in the form of blocks for heterogeneities of the next rank. A simple averaging of the measured geophysical parameters can lead to distorted ideas about the structure of the medium and its evolution [3]. One of the fundamental mining problems, which are traditionally referred to the problems of geomechanics, is the development of theoretical and experimental methods for studying the structure and condition of rock masses in order to predict and prevent catastrophic phenomena during mining. This problem is compounded by the fact that the rock massif is under direct or indirect technogenic impact, which leads to significant 
unsteadiness of both the structure and the state of the mass [4]. When conducting mining operations in highly stressed rock massifs, technogenic seismicity is manifested, the forecasting and prevention issues of which receive a lot of attention in all countries with a developed mining industry. An important role here belongs to the short-term forecast; the methodology for selecting criteria for it is still a problem, both in mining and in seismology [5]. In the framework of the Institute of Mining of the Siberian Branch of Russian Academy of sciences, important results have been achieved on the study of the state of a rock massif in the framework of nonlinear geomechanics [6] using geophysical methods with a resolution to detect the nucleation and decay of self-organizing structures [7]. In [7], studies were conducted aimed at developing criteria for the spatial-temporal complex active and passive seismic and electromagnetic monitoring to prevent destructive dynamic phenomena based on six-year seismological monitoring data carried out by the mountain impact service at the Tashtagol underground mine and the experience gained use of the system of induction electromagnetic spatial-temporal monitoring developed at the Institute of geophysics of Ural Branch of Russian Academy of sciences for arrays of various material composition before and after mass explosions.

\section{Theory, Research Methods}

We analyzed the morphology of the structural features of disintegration zones before a strong dynamic phenomenon. During the next cycle of electromagnetic observations at the Tashtagol mine in August 2007 On August 9, a mountain impact occurred with an energy of $\log E=6.9$ in the pillar, located in the orth 3 alignment at a level of $16 \mathrm{~m}$ below the soil of horizon -280, Figure 1 .

Three days before a mountain shock, in the orth 3 (Figure 1, Figure 2), in the geoelectric sections of the soil, sub vertical discrete structures are found in which disintegration zones are combined. These structures appeared in the resonance mode at different frequencies and only at one frequency for each of the unit vectors. We discovered the same phenomenon earlier in one day at Estyuninskaya mine and SUBR mine 15 [8]. The appearance of these structures of sub vertical morphology is a precursor of a strong dynamic phenomenon; however, to determine the place and magnitude of an event, it is necessary to have information on the state of the unit arrays and their belonging to the corresponding ranks on the stability of the array, as was done in [9].

At present, theoretical results on modeling the electromagnetic and seismic fields in a layered medium with hierarchical inclusions are in demand. Modeling algorithms were constructed in the electromagnetic case for 3D heterogeneity, in the seismic case for 2D heterogeneity [10] [11]. It is shown that with an increase in the degree of hierarchy of the medium, the degree of spatial non-linearity of the distribution of components of the seismic and electromagnetic fields increases, which corresponds to the detailed monitoring experiments in shock hazardous mines of the Tashtagolsky mine and SUBR. The constructed theory 

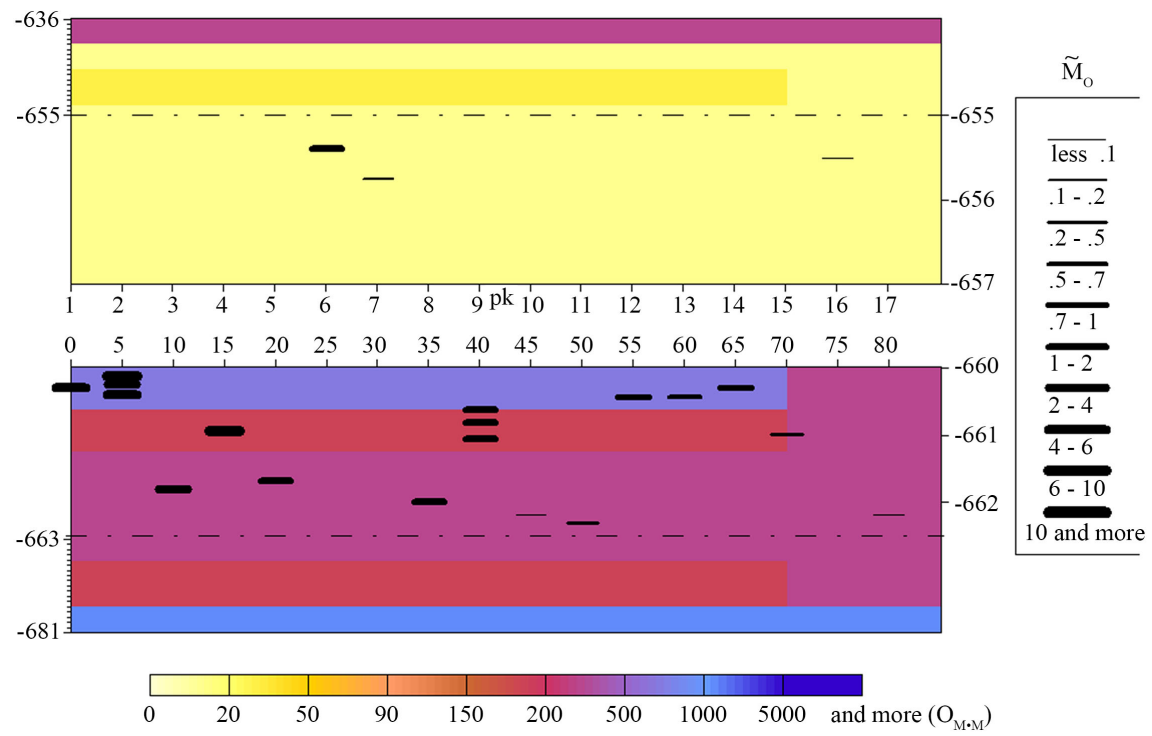

Figure 1. Geoelectrical section of a block-layered section of rock massif with inclusions of the second rank in a rock burst mine Tashtagol (the upper half of the section corresponds to the roof structure, the lower - to the floor) Morphology of anomaly stressed objects. Legend: $==\mathrm{M} 0 \times \mathrm{L} 0 \times 103, \mathrm{M} 0$ is the coefficient by which the moment of the electric current line is multiplied, equivalent in field to the influence of the zone of geoelectric heterogeneity, and which is proportional to the ratio of the difference in conductivities in the enclosing medium and in inclusion to the conductivity in the enclosing medium, L0, the length of the current line, the resistance of the enclosing section is given in ohm.m. The vertical values are in $\mathrm{m}$ (absolute marks), the horizontal is the length of the output in pickets $(\mathrm{pc})$ and meters.

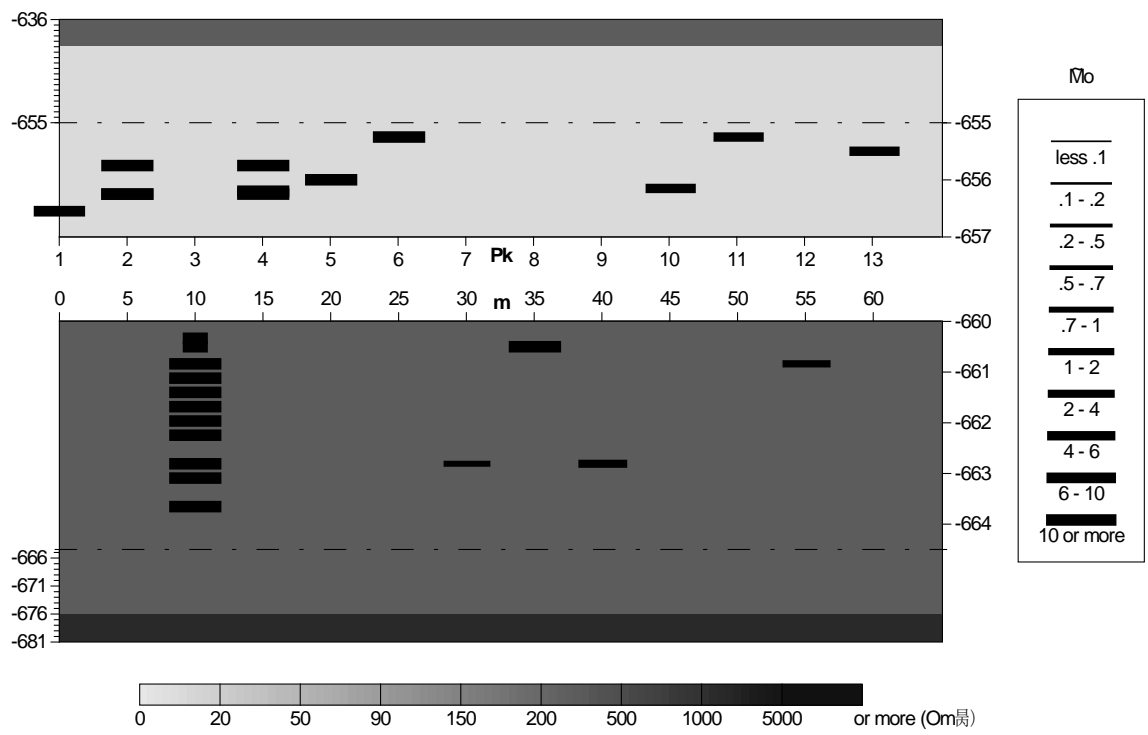

Figure 2. Geoelectric section along the profile of ort3, horizon-210, North-Western section, August 7, 2007, frequency $5.08 \mathrm{kHz}$. The legend is the same as in Figure 1.

demonstrated how complicated the process of complexion methods using an electromagnetic and seismic field is to study the response of a medium with a hierarchical structure. This problem is linked with the formulation and solution 
of the inverse problem for the propagation of electromagnetic and seismic fields in such complex media. In [12] [13], the problem of constructing an algorithm for solving the inverse problem using the equation of the theoretical inverse problem for the 2D Helmholtz equation was considered. Explicit equations of the theoretical inverse problem are written out for the cases of scattering of an electromagnetic field ( $\mathrm{E}$ and $\mathrm{H}$ polarization) and scattering of a linearly polarized elastic wave in a layered conducting and elastic medium with a hierarchical conducting or elastic inclusion, which are the basis for determining the contours of misaligned inclusions 1-rank of hierarchical structure. Obviously, when solving the inverse problem, monitoring systems configured to study the hierarchical structure of the environment should be used as the initial monitoring data. On the other hand, the more complex the medium, the each wave field brings its own information about its internal structure; therefore, the interpretation of the seismic and electromagnetic fields must be carried out separately, without mixing these databases.

\section{Results}

\subsection{Two-Dimensional Sound Diffraction Modeling Abnormally Stressed Heterogeneity of a Hierarchical Type, Located in an $\mathrm{N}$-Layer Elastic Medium}

In [14], an algorithm for modeling sound diffraction by a two-dimensional elastic hierarchical inclusion located in the J-th layer of an N-layer medium is described $G_{S p, j}\left(M, M^{0}\right)$ the function of the source of the seismic field, the boundary-value problem for which was formulated in [14]; $k_{1 j i}=\omega^{2}\left(\sigma_{i j} / \lambda_{i j}\right)$ wave number for a longitudinal wave, in the above expression, the index $j i$ means belonging to the properties of the medium inside the heterogeneity, $j a$ is outside the heterogeneity, $\lambda$ is the Lame constant; $\sigma$ is the density of the medium; $\omega$ is the circular frequency; $\boldsymbol{u}=\operatorname{grad} \varphi$ vector of displacements; $\varphi^{0}$ is the potential of a normal seismic field in a layered medium in the absence of heterogeneity: $\varphi_{j i}^{0}=\varphi_{j a}^{0}$. We assume that the density of the hierarchical inclusion for all ranks 1 and the enclosing layer are the same, and the elastic parameters of the hierarchical inclusion for all ranks differ from the elastic parameters of the enclosing medium, then the system of Equations (3) can be rewritten in the form:

$$
\begin{aligned}
& \frac{k_{1 j i l}^{2}-k_{1 j}^{2}}{2 \pi} \iint_{S C l} \varphi_{l}(M) G_{S p, j}\left(M, M^{0}\right) \mathrm{d} \tau_{M}+\varphi_{l-1}^{0}\left(M^{0}\right)=\varphi_{l}\left(M^{0}\right), M^{0} \in S_{C l}, \\
& \frac{\sigma_{j i l}\left(k_{1 j i l}^{2}-k_{1 j}^{2}\right)}{\sigma\left(M^{0}\right) 2 \pi} \iint_{S C l} \varphi_{l}(M) G_{S p, j}\left(M, M^{0}\right) \mathrm{d} \tau_{M}+\varphi_{l-1}^{0}\left(M^{0}\right)=\varphi_{l}\left(M^{0}\right), M^{0} \notin S_{C l} .
\end{aligned}
$$

\subsection{Elastic Shear Wave Diffraction Modeling on an Abnormally Stressed Heterogeneity of a Hierarchical Type, Located in an N-Layer Elastic Medium}

Similarly to (1), the same process is written for modeling the propagation of an elastic transverse wave in an N-layer medium with a two-dimensional hierar- 
chical structure of an arbitrary section morphology using the integral relations written in [15].

$$
\begin{aligned}
& \frac{k_{2 j i l}^{2}-k_{2 j}^{2}}{2 \pi} \iint_{S C l} u_{x l}(M) G_{S s, j}\left(M, M^{0}\right) \mathrm{d} \tau_{M}+\frac{\mu_{j a}}{\mu_{j i l}} u_{x(l-1)}^{0}\left(M^{0}\right) \\
& +\frac{\mu_{j a}-\mu_{j i l}}{\mu_{j i l} 2 \pi} \oint_{C l} u_{x l}(M) \frac{\partial G_{S s, j}}{\partial n} \mathrm{~d} c=u_{x l}\left(M^{0}\right), M^{0} \in S_{C l}, \\
& \frac{\mu_{j i l}\left(k_{2 j i l}^{2}-k_{2 j}^{2}\right)}{\mu\left(M^{0}\right) 2 \pi} \iint_{S C l} u_{x l}(M) G_{S s, j}\left(M, M^{0}\right) \mathrm{d} \tau_{M}+u_{x(l-1)}^{0}\left(M^{0}\right) \\
& +\frac{\mu_{j a}-\mu_{j i l}}{\mu\left(M^{0}\right) 2 \pi} \oint_{C l} u_{x l}(M) \frac{\partial G_{S s, j}}{\partial n} \mathrm{~d} c=u_{x l}\left(M^{0}\right), M^{0} \in S_{C l} .
\end{aligned}
$$

$G_{S s, j}\left(M, M^{0}\right)$ - the source function of the seismic field of the problem under consideration, it coincides with the Green function written in [15] for the corresponding problem;

$k_{2 j i l}^{2}=\omega^{2}\left(\sigma_{j i l} / \mu_{j i l}\right), \quad \mu_{j i l} \neq \mu_{j a}$ shear wave number, $\sigma_{j i l}=\sigma_{j a} ; \mu$ Lame constant; $u_{x l}$ component of the displacement vector; $l=1, \cdots, L$ hierarchical level number; $u_{x l}^{0}$ component of the displacement vector of the seismic field in a layered medium in the absence of heterogeneity of the previous rank, if $l=2, \cdots, L, \quad u_{x l}^{0}=u_{x(l-1)}$, if $l=1, \quad u_{x l}^{0}=u_{x}^{0}$, which coincides with the corresponding expression for the normal field in the work [15]. It should be noted that the structure of Equations (4) coincides with the general case when the hierarchical heterogeneity has not only elastic parameters different from the parameters of the enclosing medium, but also density parameters at all ranks differ from the density parameters of the enclosing layer. The difference between these problems lies only in the values of the wave number. Thus, the response of the medium associated with the longitudinal wave is more sensitive to the region of elastic heterogeneities in the array. This should be taken into account when assessing the status of a complex organized geological environment. In [13], the problem of constructing an algorithm for solving the inverse problem using the equation of the theoretical inverse problem for the 2-D Helmholtz equation was considered. An explicit equation of the theoretical inverse problem is obtained for the cases of scattering of a linearly polarized elastic wave in a layered elastic medium with a hierarchical elastic inclusion, the density of which for all ranks is equal to the density of the containing layer. An iterative algorithm for determining the contours of misaligned inclusions of the k-th rank in a hierarchical structure is constructed with the sequential use of the solution of the direct problem of calculating the elastic field of k-1 rank. With an increase in the degree of hierarchy of the structure of the medium, the degree of spatial non-linearity of the distribution of the components of the seismic field increases, which implies the exclusion of linearization methods for creating interpretation methods. This problem is linked with the solution of the inverse problem for the propagation of the seismic field in such complex environments using explicit equations of the theoretical inverse problem. For the first time, an equation was 
written for determining the surface of an abnormally stressed inclusion in a hierarchical layered-block medium according to acoustic monitoring data. In practice, using this algorithm, according to acoustic monitoring data, we can localize the region of a possible source of a rock shock or an earthquake and estimate the degree of anomalous elastic stresses.

\section{Discussion of Results and Conclusions}

Comparing expressions (1) and (2) we can draw the following conclusions. When constructing a complex seismic-gravity model without taking into account the anomalous influence of the stress-strain state inside the inclusion, the analysis of the anomalous acoustic effect using the data on the propagation of a longitudinal wave shows that it is also more sensitive to the form of inclusion, compared with the acoustic effect on the propagation of a transverse wave. However, it follows from these expressions that the influence of the elastic parameters in the host medium in the seismic model cannot be neglected, and they, when interpreted, affect the values of the desired anomalous densities. If these values are used in constructing the gravitational density model, then these density values will not reflect the material composition of the analyzed medium. When constructing an anomalously stressed geomechanical model without taking into account the anomalous effect of density heterogeneities within the inclusion, an analysis of the anomalous acoustic effect using data on the propagation of a shear wave shows that it is also more sensitive to the form of inclusion, compared with the acoustic effect on the propagation of a longitudinal wave. However, it follows from these expressions that the influence of the density parameters in the host medium in the seismic model cannot be neglected, and when interpreted, they affect the values of the desired anomalous elastic parameters that cause the anomalous stress state. If these values are used in the construction of the geomechanical model, then these values of the elastic parameters will not reflect the stress state of the analyzed medium. It is shown that with an increase in the degree of hierarchy of the medium, the degree of spatial non-linearity of the distribution of components of the seismic and electromagnetic fields increases, which corresponds to the detailed monitoring experiments in shock-hazardous mines of the Tashtagolsky mine and SUBR. The constructed theory demonstrated how complicated the process of complexion methods using an electromagnetic and seismic field to study the response of a medium with a hierarchical structure. This problem is linked with the formulation and solution of the inverse problem for the propagation of electromagnetic and seismic fields in such complex media. In [13], the problem of constructing an algorithm for solving the inverse problem using the equation of the theoretical inverse problem for the 2D Helmholtz equation was considered. Using the theory of solving the inverse problem, one can trace the migration of zones of abnormal stresses, their increase or decrease due to cyclic explosive influences during mining of the array, and also assess the possible risk of high-energy dynamic phenomena in the array. 


\section{Conflicts of Interest}

The authors declare no conflicts of interest regarding the publication of this paper.

\section{References}

[1] Ebeling, V. (1979) The Formation of Structures during Irreversible Processes. Mir, Moscow.

[2] Glensdorf, P. and Prigogine, I. (1973) Thermodynamic Theory of Structure, Stability and Fluctuations. Mir, Moscow.

[3] Sadovskiy, M., Bolkhovitinov, L. and Pisarenko, V. (1987) Deformation of the Geophysical Environment and the Seismic Process. Nauka, Moscow.

[4] Hachay, O.A., Vloch, N.P., Novgorodova, E.N., Khachai, A.Y. and Khudyakov, S.V. (1987) Three-Dimensional Electromagnetic Monitoring of the State of a Rock Massif. Physics of the Earth, No. 2, 85-92.

[5] Kozyrev, A.A., Savchenko, S.N., Panin, V.I. and Maltsev, V.A. (2001) Features of the Forecast and Prevention of Powerful Dynamic Phenomena in Natural-Technical Systems. Proceedings of the International Conference on Geodynamics and Stress State Bowels of the Earth, Novosibirsk, Russia, 2-4 October 2001, 326-334.

[6] Kurlenja, M.V. and Oparin, V.N. (1999) Current Problems of Nonlinear Geomechanics. Proceedings of the International Conference on Geodynamics and Stress State of the Bowels of the Earth, Novosibirsk, 6-10 July 1999, 5-20.

[7] Hachay, O.A. (2006) The Problem of Studying the Transition Process of Redistribution of the Stressed and Phase States of the Array between Strong Technogenic Effects. Mining Information and Analytical Bulletin, No. 5, 109-115.

[8] Hachay, O.A., Novgorodova, E.N., Khachay, O.Y., Kononov, A.V. and Nasedkin, V.G. (2007) The Results of Geophysical and Geological Research at the Estyuninsky Mine. Proceedings of the Fourth Scientific Readings in Memory of Yu. P. Bulashevitch on Deep Structure, Geodynamics, Thermal Field of the Earth, Interpretation of Geophysical Fields, Yekaterinburg, 2-6 July 2007, 197-199.

[9] Hachay, O.A. (2007) The Study of the Instability Development in a Rock Massif Using the Method of Active Electromagnetic Monitoring. Physics of the Earth, No. 4, 65-70.

[10] Hachay, O.A., Khachai, O.Y. and Khachay, A.Y. (2015) New Methods of Geoinformatics of Monitoring Wave Fields in Hierarchical Environments. Geoinformatics, No. 3, 45-51.

[11] Hachay, O.A., Khachai, O.Y. and Khachai, A.Y. (2016) New Methods of Geoinformatic for the Integration of Seismic and Gravitational Fields in Hierarchical Environments. Geoinformatics, No. 3, 25-29.

[12] Hachay, O.A. and Khachay, A.Y. (2016) Determination of the Surface of Anomalously Stressed Inclusions in a Hierarchical Layered-Block Medium According to Acoustic Monitoring. Mining Information and Analytical Bulletin, No. 4, 354-356.

[13] Hachay, O.A., Khachai, O.Y. and Khachai, A.Y. (2017) On the Inverse Problem of Active Electromagnetic and Acoustic Monitoring of a Hierarchical Geological Environment. Geophysical Research, 18, 71-84. https://doi.org/10.21455/gr2017.4-6

[14] Hachay, O.A. and Khachai, A.Y. (2011) On the Integration of Seismic and Electromagnetic Active Methods for Mapping and Monitoring the State of Two-Dimensional Heterogeneities in an N-Layer Medium. Bulletin of SUSU. Series Computer Technology, management, Electronics, No. 2, 49-56. 
[15] Hachay, O.A. and Khachai, A.Y. (2013) Modeling of Electromagnetic and Seismic Fields in Hierarchically Heterogeneous Media. Bulletin of SUSU, Series Computational Mathematics and Computer Science, 2, 48-55.

https://doi.org/10.14529/cmse130204 